\title{
Temperature dependent Synthesis of Zinc Sulfide Nanocrystals
}

\author{
KAMAL BERA $^{1 *}$, SATYAJIT SAHA ${ }^{1}$ and PARESH CHANDRA JANA ${ }^{1}$ \\ Department of Physics and Technophysics, Vidyasagar University, Paschim Medinipur, 721102, India. \\ ${ }^{*}$ Corresponding author E-mail: kamal.phy87@gmail.com \\ http://dx.doi.org/10.13005/ojc/340363
}

(Received: May 07, 2017; Accepted: June 01, 2018)

\begin{abstract}
Chemical reduction route is preferred to prepare $\mathrm{ZnS}$ semiconductor nanoparticles at three different temperatures without any subsequent heat treatment. The grown nanoparticles are investigated using X-ray diffraction (XRD), Transmission electron microscopy (TEM), Scanning electron microscopy (SEM), Fourier Transform Infrared (FTIR) Spectroscopy, Ultraviolet-Visible (UV-Vis) Absorption and Photoluminescence (PL) spectroscopy for structural and optical analysis. From the investigation, it is seen that the crystal size appears to increase with an increase in synthesis temperature. The cubic crystal structure is shown in XRD analysis. The UV-Vis spectrum shows the increase in the band gap which supports quantum confinement effect. The PL spectrum of ZnS nanocrystals displays a visible violet spectrum. The FTIR spectra determine the absorbance and nature of bonds present in samples.
\end{abstract}

Keywords: ZnS nanomaterials, Temperature Effect, Structural properties, Optical properties.

\section{INTRODUCTION}

In the modern age of science, semiconductor nanostructure material has gained much popularity due to their novel optical and electrical properties, which is arising from quantum confinement effects ${ }^{1-3}$. As one of the most important II-IV semiconductors, $\mathrm{ZnS}$ has found in many applications like fluorescence probe4, phosphors ${ }^{5-6}$, pharmaceutics ${ }^{7-8}$, Light Emitting Diode (LED) ${ }^{9}$, electroluminescenc ${ }^{10}$ and non-linear optical devices ${ }^{11}$.

Synthesis temperature has an important role in controlling particle shape and size ${ }^{12}$. As the growth of particle are affected by the kinetics and thermodynamics of the process. Temperature can influence the particle growth mechanism i.e., Ostwald ripening process through interfacial energy, diffusion and growth rate coefficients, and equilibrium solubility ${ }^{13}$.

However, its properties are critically dependent on synthesis technique. There are various synthesis method ZnS nanocrystals, such as the single source molecular precursor ${ }^{14}$, solvothermal synthesis ${ }^{15-16}$, sputtering ${ }^{17}$, ultrasonic irradiation18 and so on. 
Our aim is to explore the possible temperature effect on the morphological, structural and optical characteristic of ZnS nanostructure synthesized by chemical reduction route ${ }^{19}$.

\section{MATERIALS AND METHODS}

For the synthesis of ZnS nanoparticle analytical grade reagents were used without any further purification. Anhydrous $\mathrm{ZnCl}_{2}(1362.8 \mathrm{mg})$, Sulfur powder (320.6 mg) and $\mathrm{NaBH}_{4}(378.3 \mathrm{mg})$ were taken to grow different samples. The reaction bath temperatures were kept at $38{ }^{\circ} \mathrm{C}, 50{ }^{\circ} \mathrm{C}$ and $60{ }^{\circ} \mathrm{C}$ respectively to grow various sample. The grown sample was named as ZnS 1, ZnS 2, ZnS 3 respectively. $\mathrm{NaBH}_{4}$ worked as a reducing agent. The whole reaction was held on a magnetic stirrer, stirring at a fixed speed for three hours. The as grown sample filtered, washed and dried for microstructural and optical study. The TEM micrographs of as grown nano sample were produced using JEOL-JEM-200 operating at 200kV. Also, SAED pattern were taken. The surface topography and composition was acquired using ZESIS Gemini2 FESEM instrument.

The Rigaku MiniFlex-II X-ray diffractometer was used in XRD measurement. The absorption measurement is performed in ShimadzuPharmaspec-1700 UV-Vis. The Hitachi-F7000-FL spectrophotometer measures the photoluminescence spectra. With the help of Perkin Elmer Spectrum Two IR Spectrometer FTIR analysis was done.

\section{RESULTS AND DISCUSSIONS}

\section{Morphological Observation}

The TEM, SAED and SEM images are shown in Fig. 1-3 of each sample synthesized at $38^{\circ} \mathrm{C}, 50^{\circ} \mathrm{C}$ and $60^{\circ} \mathrm{C}$ respectively. Images evidently indicate the nanoparticles formation. It shows as temperature increases crystal size also increase. This is enhanced due to Ostwald ripening process taking place at a higher temperature. As the rate of reduction of sulfur increases with temperature the number of nucleation centers increases at higher temperature.

From the SAED patterns, it is seen that at $38{ }^{\circ} \mathrm{C}$ growth temperature the sample is likely to be in the single crystalline structure. The polycrystalline structure is evident at higher synthesis temperature.

Figures $1 b, 2 b, 3 b$ shows the SEM micrographs of synthesized ZnS nanoparticles with spherical shaped.It is evident that crystal size increases with growth temperature. Sample grown at $38{ }^{\circ} \mathrm{C}$ temperature shows small sized crystal. With increase of growth temperature particles get agglomerated and plate-shapedlarge clusters form.

\section{X- Ray diffraction measurements}

XRD peaks corresponds to (111), (220), (311) plane matched cubic zinc blende structure (JCPDS No. 05-0566), confirm pure ZnS nanocrystal. With the increase of growth temperature, the intensity corresponding to (220) and (311) plane is reduced.

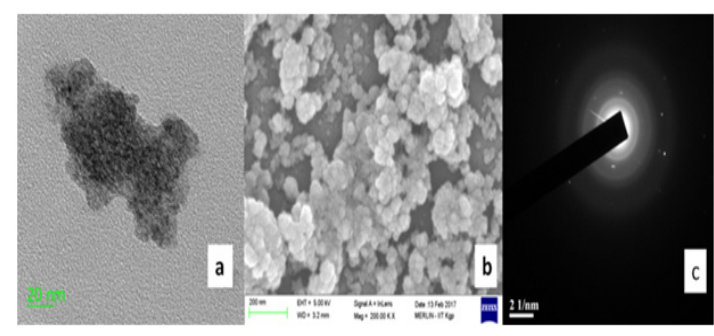

Fig. 1. (a) TEM, (b) SEM (c) SAED images of as synthesized ZnS 1 sample

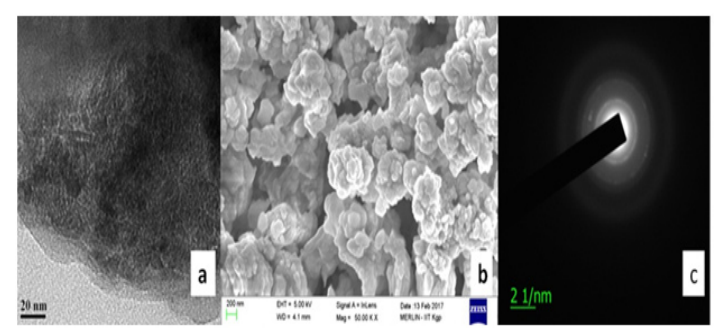

Fig. 2. (a) TEM, (b) SEM (c) SAED images of as synthesized ZnS 2 sample

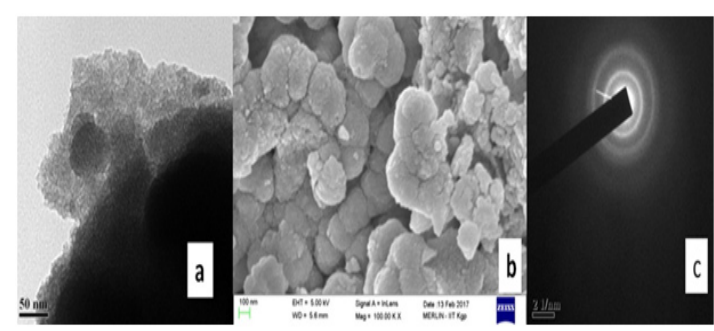

Fig. 3. (a) TEM, (b) SEM (c) SAED images of as synthesized ZnS 3 sample 


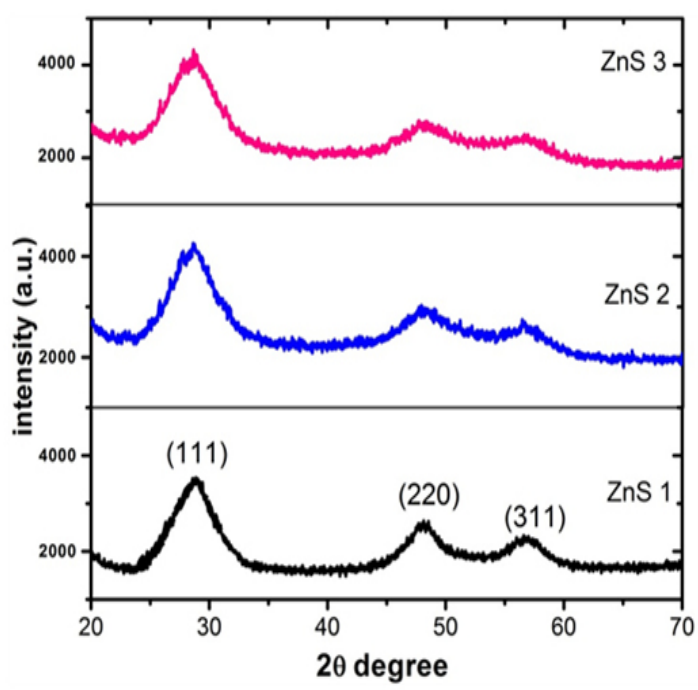

Fig. 4. XRD pattern of ZnS nanomaterials

\section{UV-Visible Measurements}

Figure 5 shows the UV-Vis absorption plot of as-synthesized samples in the wavelength range 200-700 $\mathrm{nm}$ at room temperature. The band gap value was estimated from Tauc plot (Fig. 6). The values of calculated band gap are about $3.74 \mathrm{eV}$, $3.96 \mathrm{eV}$, and $4.45 \mathrm{eV}$ which are comparable to the standard energy band gap of ZnS i.e., $3.68 \mathrm{eV}$. It is observed that energy band gap decreases with increasing temperature. This supports the increment in crystal size with temperature and also confirms that temperature plays an important in the optical properties of synthesized ZnS nanocrystals.

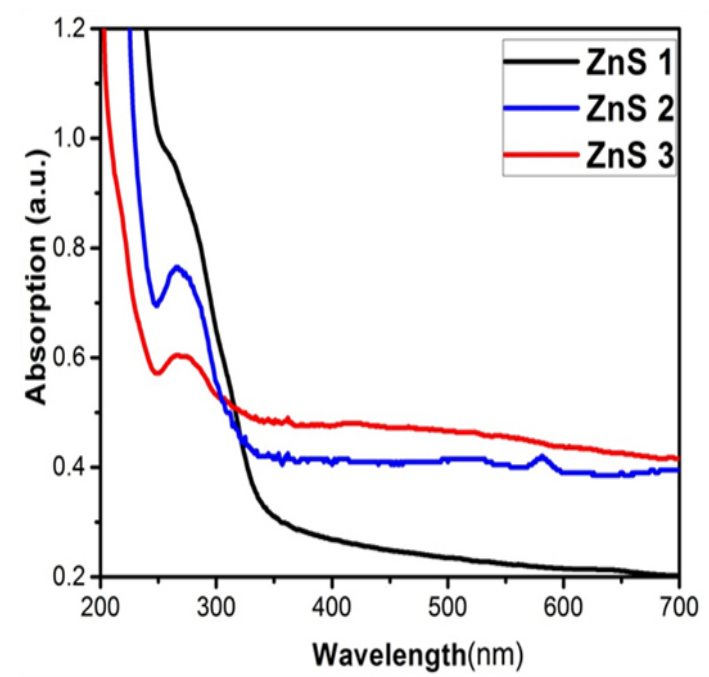

Fig. 5. UV-VIS absorption spectra of different sample

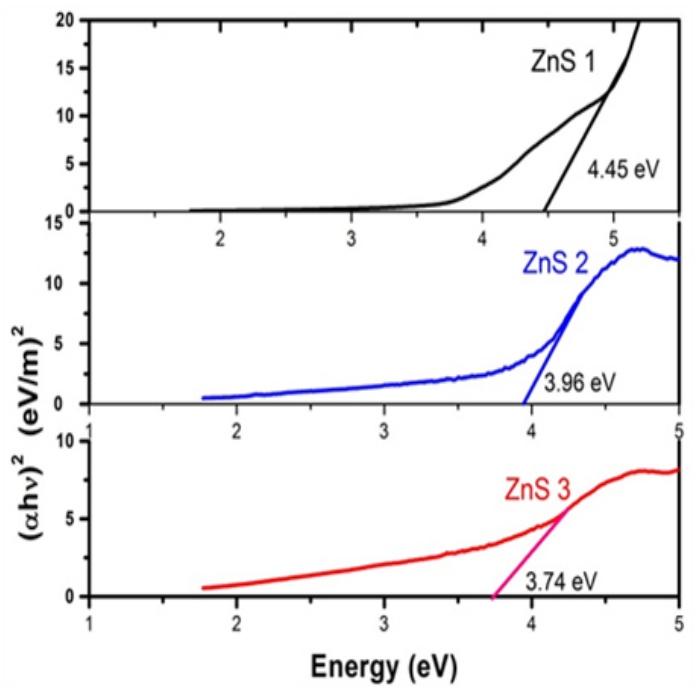

Fig. 6. Determination of band gap energy

\section{Fluorescence measurements}

Figure 7 shows the photoluminescence spectrum of as-synthesized ZnS nanoparticles to investigate luminescence properties. The PL intensity increases with increase in growth temperature. This is due to increase in particle size ${ }^{20}$. There is also peak broadening for all sample. This is due to non-uniform particle distribution and it increases with growth temperature.

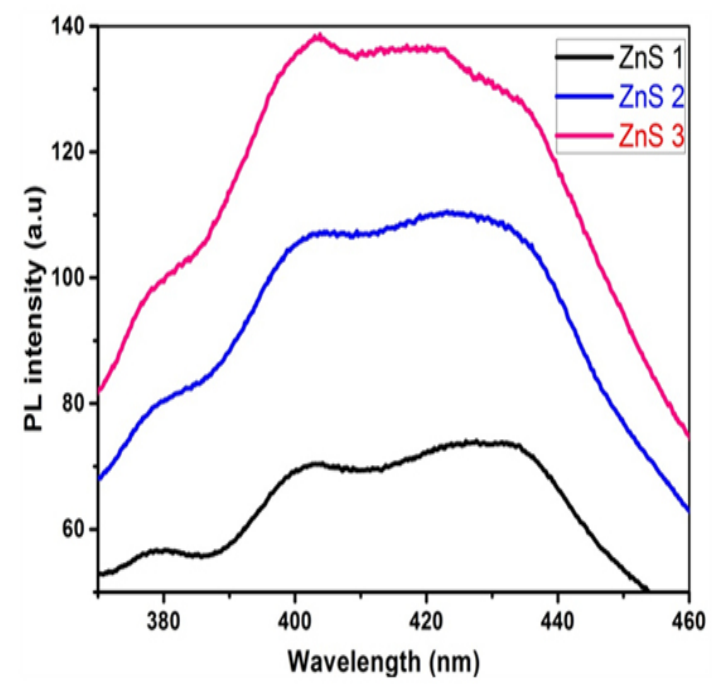

Fig. 7. The fluorescence spectra of various samples

By decryption of fluorescence spectra of sample growth at $38^{\circ} \mathrm{C}$ temperature (Fig. 8) we get an accurate position of fluorescence peak at 378.9, $400.5,416.8,433.7 \mathrm{~nm}$. The peak at $378.9 \mathrm{~nm}$ is due 
to band edge luminescence. Peaks at $400.5,416.8$ and $433.7 \mathrm{~nm}$ are due to surface state arising from vacancies and interstitials of sulfur and zinc atom ${ }^{21}$.

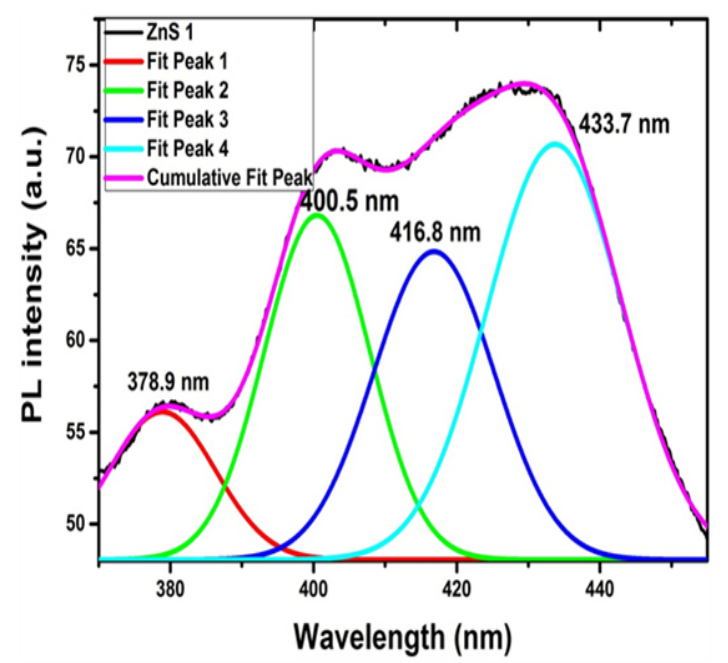

Fig. 8. Decryption of fluorescence spectra of sample growth at $38^{\circ} \mathrm{C}$

Fourier transforms infrared spectroscopy (FTIR) measurement

The FTIR spectra are carried out to analyze the composition and quality of the compound in the range $4000-500 \mathrm{~cm}^{-1}$, shown in Fig. 9. The FTIR spectra of three ZnS sample show that the transmission bands are almost similar to each other. Only some extra dips occur in the case of higher temperature.

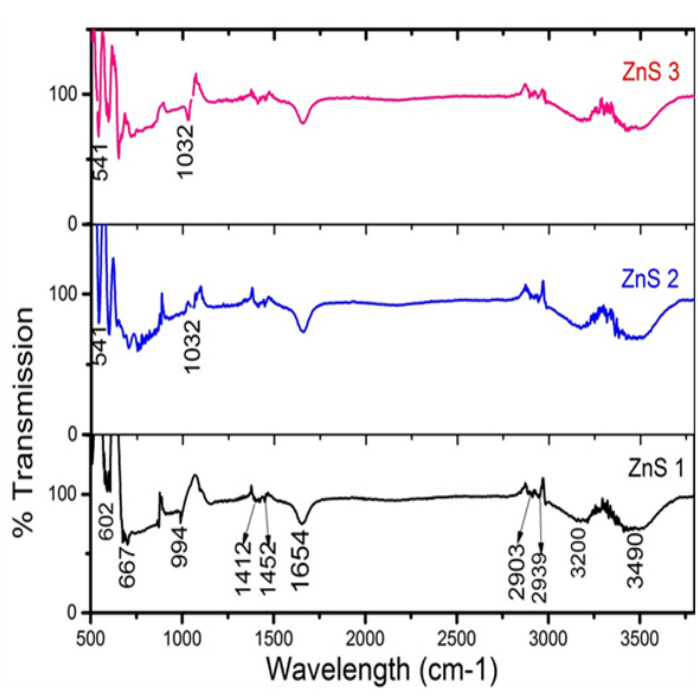

Fig. 9. FTIR spectra of ZnS nanoparticles synthesized at different temperature

The dips observed at 602 and $667 \mathrm{~cm}^{-1}$ are due to characteristic Zn-S vibration ${ }^{22}$. Dips at 1412 and 1442 are probably due to the microstructure formation of the samples ${ }^{23}$. Dips around $1654 \mathrm{~nm}$ are due to the $\mathrm{C}-\mathrm{O}$ vibrational modes arise from absorbed $\mathrm{CO}_{2}$ on the surface of the nanocrystals ${ }^{24}$. Dips at 2903 and 2939 are due to $\mathrm{CH}_{2}$ and $\mathrm{CH}_{3}$ vibrational mode ${ }^{25}$. The band around $3160-3226$, $3420-3550 \mathrm{~cm}^{-1}$ are due to the hydrogen Stretching frequency (OH stretching) of the hydroxyl group.

Table 1: Comparison of different sample

\begin{tabular}{lcccc}
\hline $\begin{array}{l}\text { Sample } \\
\text { Name }\end{array}$ & $\begin{array}{c}\text { Growth } \\
\text { Temperature } \\
\left({ }^{\circ} \mathrm{C}\right)\end{array}$ & $\begin{array}{c}\text { Bandgap } \\
(\mathrm{eV})\end{array}$ & $\begin{array}{c}\text { Particle } \\
\text { size } \\
(\mathrm{TEM})\end{array}$ & $\begin{array}{c}\text { Atomic \% } \\
\text { EDSanalysis }\end{array}$ \\
\hline ZnS 1 & 38 & 4.45 & $15 \mathrm{~nm}$ & $\begin{array}{c}\mathrm{Zn}=33.24 \\
\mathrm{~S}=66.76\end{array}$ \\
ZnS 2 & 50 & 3.96 & $40 \mathrm{~nm}$ & $\begin{array}{c}\mathrm{Zn}=45.6 \\
\mathrm{~S}=54.4\end{array}$ \\
ZnS 3 & 60 & 3.74 & $50 \mathrm{~nm}$ & $\begin{array}{r}\mathrm{Zn}=57.56 \\
\mathrm{~S}=42.44\end{array}$ \\
\hline
\end{tabular}

\section{CONCLUSION}

The temperature effects on structural, optical and chemical characteristics of ZnS nanocrystals are investigated and summarized intable 1. The XRD analysis shows cubic phase in all samples. The
TEM images suggest that crystal size increases with growth temperature. With increasing growth temperature the formation of nuclei are increased and by Ostwald ripening method the crystal size also increase. The energy gap of band decreases at higher growth temperature. It is observed that from 
SEM images that the agglomeration is prominent at higher growth temperature. Fluorescence intensity increases for the sample grown at higher temperature. This is probably owing to the excess of a constituent ion such as Zinc with respect to Sulphur.

\section{ACKNOWLEDGMENT}

Authors acknowledge UGC and DST for constant support to Physics and Techno-physics department through SAP and FIST program.

\section{REFERENCES}

1. Takagahara, T; Takeda, K. Physical Review B., 1992, 46, 15578.

2. Alivisatos, A. P. Science., 1996, 271, 933.

3. Buhro, W.E. ; Colvin, V.L. Nature materials., 2003, 2, 138-139.

4. Li, Y.; Chen, J.; Zhu, C.; Wang, L.; Zhao, D.; Zhuo, S.; Wu, Y, Spectrochimica Acta Part A: Molecular and Biomolecular Spectroscopy. 2004, 60, 1719-1724.

5. Okuda, H.; Takada, J.; Iwasaki, Y.; Hashimoto, N.; Nagao, C., IEEE Transactions on Consumer Electronics., 1990, 36, 436-443.

6. Ghrayeb, J.; Jackson, T.W.; Daniels, R.; Hopper, D.G.In AeroSense. International Society for Optics and Photonics., 1997, 97 237-248.

7. Pathakoti, K.; Hwang, H.M.; Xu, H.; Aguilar, Z.P. ; Wang, A. Journal of Environmental Sciences., 2013, 25, 163-171.

8. Kumar, P.; Maikap, S.; Singh, K.; Chatterjee, S.; Chen, Y.Y.; Cheng, H. M.; Mahapatra, R. ; Jian-Tai Qiu, J.T.;Yang, J. R. Journal of The Electrochemical Society., 2016, 163, B580-B587.

9. Kim, H.; Han, J.Y.; Kang, D.S.; Kim, S.W.; Jang, D.S.; Suh, M.; Kirakosyan, A.; Jeon, D.Y. Journal of Crystal Growth., 2011, 326, 90-93.

10. Xuan, R.-W; Xu, J.-P.; Zhang, X.-S.; Li, P.; Luo, C.-Y.;Wu, Y.-Y.; Li, L. Appl. Phys. Lett., 2011, 98, 041907.

11. $\mathrm{Xu}, \mathrm{J} . ; \mathrm{Ji}, \mathrm{W}$. Journal of materials science letters., 1999, 18, 115-117.

12. Mott, D.; Galkowski, J.;Wang, L.; Luo, J.; Zhong, C. J. Langmuir., 2007, 23, 5740-5745.
13. Madras, G.; McCoy, B.J. The Journal of chemical physics., 2003, 119, 1683-1693.

14. Barrelet, C.J.; Wu, Y.; Bell, D.C. ; Lieber, C.M. Journal of the American Chemical Society., 2003, 125, 11498-11499.

15. Ashwini, K. ; Pandurangappa, C. Optical Materials., 2014, 37, 537-542.

16. Dong, L.; Chu, Y.; Zhang, Y. Materials Letters., 2007, 61, 4651-4654.

17. Hwang, D.H.;Ahn, J.H.;Hui, K.N.;San Hui, K.;Son, Y.G. Nanoscale research letters., 2012, 7, 26.

18. Xu, J.F.; Ji, W.; Lin, J.Y.; Tang, S.H. ; Du, Y.W. Applied Physics A: Materials Science \& Processing., 1998, 66, 639-641.

19. Wang, W.; Germanenko, I. ; El-Shall, M.S. Chemistry of Materials., 2002, 14, 3028-3033.

20. Xiong, G.; Pal, U. ; Serrano, J.G. Journal of applied physics., 2007, 101, 024317.

21. Denzler, D.; Olschewski, M.; Sattler, K. Journal of applied physics., 1998, 84, 2841-2845.

22. Thangam, Y.Y.; Anitha, R. ; Kavitha, B. International Journal of Applied Science and Engineering Research., 2012, 1, 282-286.

23. Ummartyotin, S.; Bunnak, N.; Juntaro, J.; Sain, M. ; Manuspiya, H. Solid State Sciences., 2012, 14, 299-304.

24. Qadri, S.B.; Skelton, E.F.; Hsu, D.; Dinsmore, A.D.; Yang, J.; Gray, H.F. ; Ratna, B.R. Physical Review B., 1999, 60, 9191.

25. Gómez-Barojas,E.; Sánchez-Mora,E.; Castillo-Abriz, C.; Flores-Rodríguez, E. ; SilvaGonzález, R. Journal of superconductivity and novel magnetism., 2013, 26, 2337-2340. 